\title{
Impact of random urine proteinuria on maternal and fetal outcomes of pregnancy: a retrospective case-control study
}

\author{
Eun Hui Bae ${ }^{1}$, Jong Woon Kim² ${ }^{2}$ Hong Sang $\mathrm{Choi}^{1}$, Seong Kwon $\mathrm{Ma}^{1}$, and Soo Wan Kim
}

Departments of ${ }^{1}$ Internal Medicine and ${ }^{2}$ Obsterics, Chonnam National University Medical School, Gwangju, Korea

Received: January 7, 2016

Revised : April 25, 2016

Accepted: May 6, 2016

\section{Correspondence to}

Soo Wan Kim, M.D.

Department of Internal

Medicine, Chonnam National

University Medical School, 42

Jebong-ro, Dong-gu, Gwangju

61469 , Korea

Tel: +82-62-220-6271

Fax: +82-62-225-8578

E-mail: skimw@chonnam.ac.kr
Background/Aims: Proteinuria is associated with hypertension and preeclampsia in pregnancy. However, the impact of random urine proteinuria on fetal and maternal outcomes has not been established. We investigated the influence of random urine proteinuria on the clinical outcomes of pregnancy.

Methods: From January 2008 to December 2010, 2,822 patients were retrospectively studied. A total of 536 pregnant women with proteinuria in random urine and matched controls without proteinuria via propensity score matching were analyzed. Proteinuria was checked by the dipstick method.

Results: The patients' mean age was $33.0 \pm 4.7$ years, and the mean gestational age was $235.6 \pm 50.6$ days on admission. The prevalence of hypertension and chronic kidney disease was $2.4 \%(n=67)$ and $1.0 \%(n=29)$, respectively. Women with random urine proteinuria showed higher blood urea nitrogen levels and a higher incidence of hematuria. These women also had a higher incidence of preeclampsia, preterm labor, premature rupture of membranes, and intrauterine growth restriction. Proteinuria was strongly correlated with preeclampsia in both propensity score matching $(p<0.001, r=0.783)$ and unmatched whole samples $(p<0.001$, $r=0.851)$.

Conclusions: These findings suggest that random urine proteinuria is associated with preeclampsia, preterm labor, premature rupture of membrane, and intrauterine growth restriction.

Keywords: Proteinuria; Dipstick; Pre-eclampsia; Random urine; Obstetric labor, premature

\section{INTRODUCTION}

There are many reports of the harmful effects of proteinuria in pregnancy in relation to hypertension and preeclampsia [1,2]. The dipstick test, which can be used for semiquantitative determination of protein concentration in spot urine, is used as a screening test to detect significant proteinuria. Many pregnant women have random urine dipsticks positive for proteinuria, and there is minimal data on the impact of random urine proteinuria in pregnancy. The gold standard for measuring proteinuria is a 24 -hour urine sample for total protein level [3], but this method may result in delayed diagnosis and treatment or possibly a prolonged hospital stay [4]. Several studies have suggested an acceptable correlation between single-voided protein-to-creatinine ratio and 24-hour urine protein in pregnancy $[5,6]$. However, the impact of random urine proteinuria on preg- 
nancy has not yet been determined. The present study aimed to evaluate random proteinuria as a predictor for the maternal and fetal outcomes of pregnancy.

\section{METHODS}

\section{Ethics statement}

This study was approved by the Institutional Review Board (IRB) of Chonnam National University Hospital, Gwangju, Republic of Korea. The study was performed in accordance with the Helsinki Declaration of 1975, as revised in 2000. Each patient in the current study was informed about data usage for this investigation. However, because this study was a retrospective medical record-based study and the study subjects were de-identified, the IRB waived the need for written consent from the patients.

\section{Subjects}

This study retrospectively analyzed data from all the pregnant women admitted to the obstetrics department of Chonnam National University Hospital from January 2008 to December 2010. Measurement of urine protein was done by spectrophotometry (Clinitek Novus, Siemens, Munich, Germany). Patients' medical records were used to obtain demographic data; gestational age on admission; gravidity; and history of diabetes, chronic hypertension, and other medical disorders.

\section{Definitions and measurements}

Proteinuria was classified by semiquantitative measurements as dipstick positive or negative. Preeclampsia was defined as the onset of hypertension and proteinuria after 20 weeks of gestation in previously normotensive women [7]. Eclampsia was defined as the occurrence of a new-onset seizure in a patient with preeclampsia. Chronic hypertension patients were defined as patients with anti-hypertension medication or systolic blood pressure $\geq 140 \mathrm{mmHg}$ before gestational week 20 . Chronic kidney disease was defined as abnormalities of kidney structure or function, present for $>3$ months before pregnancy [8]. The estimated glomerular filtration rate (eGFR) was calculated by The Chronic Kidney Disease Epidemiology Collaboration (CKD-EPI) equation. The definition of chronic kidney disease (CKD) was tak- en from the guidelines published by the National Kidney Foundation's Kidney Disease Outcomes Quality Initiative [9]. Other adverse conditions were evaluated, consisting of maternal complications (preterm birth history, abortion, preterm labor, premature rupture of membranes [PROM]), fetal complications (intrauterine growth restriction [IUGR], intrauterine fetal death, neonatal death), and abnormal maternal laboratory testing (hemoglobin, albumin, blood urea nitrogen, hematuria). Neonatal death was defined as death before discharge from the hospital. Infant weight was deemed normal if greater than $2.5 \mathrm{~kg}$ [9]. Abortion was defined as the termination of pregnancy before 20 weeks of gestation [10].

\section{Statistical analysis}

Data are presented as mean \pm standard deviation, median (range), or number (\%). Baseline characteristics of the cohort are described using parametric methods for continuous variables and nonparametric methods for categorical variables. The Student $t$ test and Pearson chi-square test (or Fisher exact test) were used to compare baseline characteristics for categorical outcomes. The receiver operating characteristic (ROC) curve analysis was performed to investigate the diagnostic performance of any marker. To address potential sources of bias or confounding variables in this observational study, adjustments were made with propensity score matching using maternal age and gestational age.

All analyses were conducted using the SPSS version 21.o (IBM Co., Armonk, NY, USA). A paired Student $t$ test was used to analyze the comparative results.

\section{RESULTS}

A total of 2,822 pregnant women were enrolled, and 536 of them had proteinuria. The patients' mean age was $33.0 \pm 4.7$ years, gestational age was $235.6 \pm 50.6$ days, and body mass index was $25.9 \pm 4.2$ at the time of admission. Past obstetric history revealed full term deliveries in 1,609 patients (56.9\%). Hypertension had previously been diagnosed in 67 patients $(2.4 \%)$ and chronic kidney disease in 29 patients (1.0\%) (Table 1).

Eight hundred and nine women (28.6\%) had a history of an artificial abortion, and 617 women (21.9\%) had a history of a spontaneous abortion. The mean creatinine 
Table 1. Baseline characteristics of all pregnant women included in this study

\begin{tabular}{lc}
\hline Characteristic & Value \\
\hline Number & 2,822 \\
Age, yr & $33.0 \pm 4.7$ \\
Gestational age, day & $235.6 \pm 50.6$ \\
Body mass index, kg/m ${ }^{2}$ & $25.9 \pm 4.2$ \\
Obstetrical past history, \% & \\
Full term & $1,609(56.9)$ \\
Preterm (<37 weeks) & $243(8.6)$ \\
\hline Artificial abortion & $809(28.6)$ \\
\hline Spontaneous abortion & $617(21.9)$ \\
Maternal risk factor & $67(2.4)$ \\
Chronic hypertension & $38(1.3)$ \\
\hline Preeclampsia & $39(1.4)$ \\
Diabetes mellitus & $36(1.3)$ \\
Smoking & $29(1.0)$ \\
\hline Chronic kidney disease & \\
\hline
\end{tabular}

Values are presented as mean $\pm \mathrm{SD}$ or number (\%).

concentration of the 2,822 women was $0.5 \pm 0.4 \mathrm{mg} / \mathrm{dL}$, and the mean eGFR was $128 \pm 14 \mathrm{~mL} / \mathrm{min} / 1.73 \mathrm{~m}^{2}$. A total of 536 women (19.0\%) showed dipstick positive proteinuria (1+, 210 women [7.4\%]; 2+, 205 women [7.3\%]; 3+, 121 women [4.3\%]). A total of 249 women (8.8\%) showed hematuria on a urine dipstick test (Table 2).

Table 3 shows the demographic and functional data of patients with and without proteinuria after propensity score matching with age and gestational age at delivery. The proteinuria group shows a higher rate of previous preeclampsia and a lower rate of past full term deliveries. The prevalence of diabetes mellitus and CKD was higher in the proteinuria group. Serum albumin levels and eGFR were lower in the proteinuria group, and blood urea nitrogen levels, serum creatinine levels, and the incidence of hematuria was higher in the proteinuria group (Table 3).

Table 4 shows the clinical outcomes of patients with and without proteinuria. Fetal body weight trended lower in the proteinuria group. The incidence of preterm labor, PROM, IUGR, and preeclampsia was higher in the proteinuria group.

Preeclampsia was the outcome most significantly with
Table 2. Laboratory findings of all the pregnant women at the visit

\begin{tabular}{lc}
\hline Variable & Value \\
\hline Number & 2,822 \\
Hemoglobin, g/dL & $11.9 \pm 1.6$ \\
Albumin, g/dL & $3.5 \pm 0.4$ \\
Blood urea nitrogen, mg/dL & $8.5 \pm 3.0$ \\
Creatinine, mg/dL & $0.5 \pm 0.4$ \\
eGFR, mL/min/1.73 $\mathrm{m}^{2}$ & $128 \pm 14$ \\
Urine protein & $536(19.0)$ \\
$1+$ & $210(7.4)$ \\
$2+$ & $205(7.3)$ \\
$3+$ & $121(4.3)$ \\
Hematuria & $249(8.8)$ \\
\hline
\end{tabular}

Values are presented as mean \pm SD or number (\%). eGFR, estimated glomerular filtration rate.

proteinuria (Pearson correlation, correlation coefficient $=85 \%, p<0.001)$. Thus, we performed ROC analysis of proteinuria for the predictive value of preeclampsia. The area of the ROC curve was $0.783(p<0.001)$ in propensity score matching samples (Fig. $1 \mathrm{~A})$ and $0.851(p<$ 0.001) in unmatched whole samples (Fig. 1B).

Table 5 shows the clinical outcomes of patients before and after gestational week 20 showing proteinuria. Fetal body weight was significantly lower in the proteinuria before gestational week 20 group. Abortion and neonatal fetal death rate also showed significantly higher in the proteinuria before gestational week 20 , but preeclampsia was higher in the proteinuria after gestational week 20 group.

\section{DISCUSSION}

This study shows that approximately one in five women have dipstick positive proteinuria at some time during their pregnancy. Our data suggest that random urine proteinuria during pregnancy was correlated with preterm labor, PROM, IUGR, and preeclampsia. Some studies have found that maternal and perinatal morbidity and mortality increased with the amount of proteinuria [11]. Several methods are available for measuring proteinuria, but 24-hour urine protein excretion has long been 
Bae EH, et al. Impact of proteinuria on pregnancy

Table 3. Comparison of demographic and functional data between two groups

\begin{tabular}{|c|c|c|c|}
\hline Variable & No proteinuria $(n=536)$ & Proteinuria $(\mathrm{n}=536)$ & $p$ value \\
\hline \multicolumn{4}{|l|}{ Obstetrical past history, \% } \\
\hline Full term & $248(46.3)$ & $172(32.1)$ & $<0.001$ \\
\hline Preterm (<37 weeks) & $45(8.4)$ & $42(7.8)$ & 0.748 \\
\hline Artificial arbortion & $171(31.9)$ & $150(28.0)$ & 0.520 \\
\hline Spontaneous arbortion & $106(19.8)$ & $123(22.9)$ & 0.493 \\
\hline Preeclampsia & $4(0.7)$ & $19(3 \cdot 5)$ & 0.002 \\
\hline \multicolumn{4}{|l|}{ Maternal risk factor } \\
\hline Hypertension & $13(2.4)$ & $24(4 \cdot 5)$ & 0.219 \\
\hline Diabetes mellitus & $5(0.9)$ & $14(2.6)$ & 0.037 \\
\hline Smoking & $7(1.3)$ & $10(1.9)$ & 0.463 \\
\hline Chronic kidney disease & $1(0.2)$ & $28(5.2)$ & 0.004 \\
\hline \multicolumn{4}{|l|}{ Lab findings } \\
\hline Hemoglobin, g/dL & $11.9 \pm 1.4$ & $12.1 \pm 1.8$ & 0.042 \\
\hline Albumin, g/dL & $3.5 \pm 0.4$ & $3.3 \pm 0.5$ & $<0.001$ \\
\hline Blood urea nitrogen, mg/dL & $8.0 \pm 2.5$ & $10.5 \pm 4.1$ & $<0.001$ \\
\hline Creatinine, g/dL & $0.5 \pm 0.3$ & $0.6 \pm 0.4$ & $<0.001$ \\
\hline $\mathrm{eGFR}, \mathrm{mL} / \mathrm{min} / 1.73 \mathrm{~m}^{2}$ & $130.0 \pm 13.0$ & $120.9 \pm 19.0$ & $<0.001$ \\
\hline Hematuria & $29(5.4)$ & $102(19.0)$ & $<0.001$ \\
\hline
\end{tabular}

Values are presented as number (\%) or mean \pm SD.

eGFR, estimated glomerular filtration rate.

Table 4. Clinical outcomes of proteinuria

\begin{tabular}{lccc}
\hline Variable & No proteinuria $(\mathrm{n}=536)$ & Proteinuria $(\mathrm{n}=536)$ & $p$ value \\
\hline Fetal body weight & $2,458 \pm 912$ & $2,360 \pm 974$ & 0.089 \\
Full term & $240(44.8)$ & $234(43.7)$ & 0.712 \\
Preterm (<37 weeks) & $257(47.9)$ & $255(47.6)$ & 0.903 \\
Preterm labor & $65(12.1)$ & $105(19.6)$ & 0.001 \\
Abortion & $14(2.6)$ & $15(2.8)$ & 0.851 \\
Neonatal death & $38(7.1)$ & $46(8.6)$ & 0.363 \\
PROM & $74(13.8)$ & $123(22.9)$ & $<0.001$ \\
IUGR & $48(9.0)$ & $78(14.6)$ & 0.004 \\
IUFD & $11(2.1)$ & $19(3.5)$ & 0.138 \\
Preeclampsia & $14(2.6)$ & $222(41.4)$ & 0.001 \\
Eclampsia & 0 & $2(0.4)$ & 0.157 \\
GDM & $20(3.7)$ & $28(5.2)$ & 0.237 \\
\hline
\end{tabular}

Values are presented as mean \pm SD or number $(\%)$.

PROM, preterm rupture of membrane; IUGR, intrauterine growth restriction; IUFD, intrauterine fetal death; GDM, gestational diabetes mellitus. 

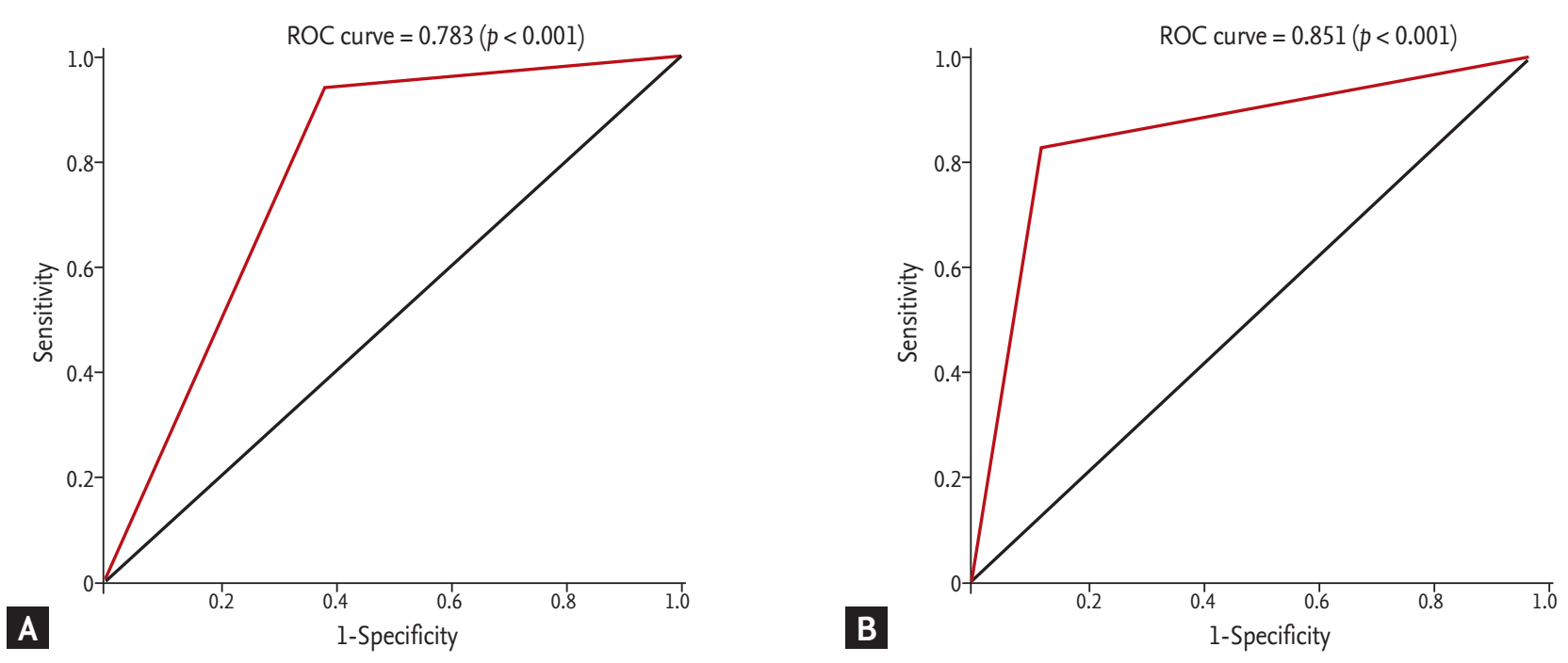

Figure 1. (A) Propensity score matching. (B) Unmatched whole samples. ROC, receiver operating characteristic.

Table 5. Clinical outcomes between before and after GW 20 showing proteinuria

\begin{tabular}{lccr}
\hline Variable & Proteinuria before GW 20 $(\mathrm{n}=54)$ & Proteinuria after GW 20 $(\mathrm{n}=482)$ & $p$ value \\
\hline Fetal body weight & $1,740 \pm 1,336$ & $2,425 \pm 908$ & 0.001 \\
Full term & $16(29.6)$ & $216(44.8)$ & 0.021 \\
Preterm (<37 weeks) & $20(37.0)$ & $235(48.8)$ & 0.066 \\
Preterm labor & $2(8.0)$ & $63(13.1)$ & 0.395 \\
Abortion & $15(27.8)$ & 0 & $<0.001$ \\
Neonatal death & $18(33.3)$ & $29(6.0)$ & $<0.001$ \\
PROM & $7(13.0)$ & $67(13.9)$ & 0.521 \\
IUGR & $5(9.3)$ & $71(14.7)$ & 0.188 \\
IUFD & 0 & $19(3.9)$ & 0.128 \\
Preeclampsia & $10(18.5)$ & $211(43.8)$ & $<0.001$ \\
Eclampsia & 0 & $2(0.4)$ & 0.808 \\
GDM & $2(3.7)$ & $26(5.4)$ & 0.448 \\
\hline
\end{tabular}

Values are presented as mean \pm SD or number $(\%)$.

GW, gestational week; PROM, preterm rupture of membrane; IUGR, intrauterine growth restriction; IUFD, intrauterine fetal death; GDM, gestational diabetes mellitus.

regarded as the gold standard. However, this test has some disadvantages such as inconvenience for patients, inaccuracy due to incomplete collection, cost, and delay of diagnosis and management, which makes its wide use difficult for clinicians. For this reason, many investigators have explored simpler and more convenient diagnostic methods to quantify proteinuria. The urine dip- stick test is one of the most commonly used screening tests because of its simplicity and low cost. Nevertheless, this method has high rates of false positive and false negative results associated with fluctuations throughout the day due to water intake, exercise, diet, posture, or improperly trained laboratory staff [12-14]. Therefore, some investigators have explored other means of quan- 
tifying proteinuria in a shorter time period and they suggest a 2-hour collection, 4-hour collection, or using the protein-creatinine ratio [15-17]. The protein to creatinine ratio of a single sample has been shown to correlate significantly with a 24-hour collection for pregnant patients with protein values of less than $1 \mathrm{~g}$ in 24 hours, but not for those with protein values above $1 \mathrm{~g}$, in which the variation between the samples was increased $[18,19]$. However, those studies evaluated the accuracy of dipstick testing for proteinuria compared to 24-hour urine collection and did not evaluate clinical outcomes. It has been recently reported that urine dipstick test results were closely related to urine creatinine concentration, and postpartum urine samples had significantly lower urine creatinine concentration compared to antepartum urine samples. It was likely to show a false positive result in concentrated urine samples with higher urine creatinine concentration for prediction of preeclampsia [20]. Unfortunately, our data did not consider urine creatinine concentration or urine osmolality.

Preeclampsia is a serious complication of pregnancy, and it is vital to diagnose the condition as early as possible. Preeclampsia is defined as the development of hypertension or proteinuria prior to week 20 or the development of both after week 20 in a woman previously having normal blood pressure [21]. Proteinuria is a defining dysfunction of preeclampsia, the degree of which may fluctuate widely over any 24 -hour period due to the circadian variation of urinary albumin excretion $[22,23]$. Some researchers have concluded that the random urine protein to creatinine ratio is not a good predictor of significant proteinuria in patients with preeclampsia $[24,25]$. Other studies have shown that urine dipstick for protein results correlate poorly with 24 -hour urine samples for differentiating patients with no disease or severe disease $[15,26]$. The results of our study indicate that random urine proteinuria is highly predictive for preeclampsia. This discrepancy with previous study results might be explained by the fact that our center is a university hospital and pregnant women with false-positive random urine dipstick protein tests might have been screened in private clinics before visiting our hospital. Moreover, our study showed proteinuria after gestational week 20 highly associated with preeclampsia compared to proteinuria before gestational week 20 group. However, the pregnant women who showed proteinuria before gesta- tional week 20 associated with lower fetal body weight, higher abortion and neonatal death rate. Those results may be affected by known CKD women who were included in the proteinuria before gestational week 20 group.

The group with random urine positive for proteinuria also showed lower serum albumin values, lower eGFR, and a higher incidence of hematuria. These laboratory findings can explain poor fetal outcomes such as IUGR.

In conclusion, random urine proteinuria during pregnancy was correlated with preterm labor, PROM, and IUGR, and was also highly predictive for preeclampsia.

\section{KEY MESSAGE}

1. During pregnancy, about $20 \%$ showed random urine proteinuria.

2. Random urine proteinuria is associated with preeclampsia, preterm labor, premature rupture of membrane, and intrauterine growth restriction.

\section{Conflict of interest}

No potential conflict of interest relevant to this article was reported.

\section{Acknowledgments}

This research was supported by Basic Science Research Program through the National Research Foundation of Korea (NRF) funded by the Ministry of Science, ICT and future Planning (2016R1A2B4007870), and by the Pioneer Research Center Program through the National Research Foundation of Korea funded by the Ministry of Science, ICT \& Future Planning (2014M3 $\mathrm{Cl}_{3} \mathrm{~A}_{3} \mathrm{O}_{303} \mathrm{O}$ ).

\section{REFERENCES}

1. Martins-Costa SH, Vettorazzi J, Valerio E, et al. Protein creatinine ratio in random urine sample of hypertensive pregnant women: maternal and perinatal outcomes. Hypertens Pregnancy 2011;30:331-337.

2. Wikstrom AK, Wikstrom J, Larsson A, Olovsson M. Random albumin/creatinine ratio for quantification of proteinuria in manifest pre-eclampsia. BJOG 2006;113:930- 
934.

3. Lambers Heerspink HJ, Brantsma AH, de Zeeuw D, et al. Albuminuria assessed from first-morning-void urine samples versus 24-hour urine collections as a predictor of cardiovascular morbidity and mortality. Am J Epidemiol 2008;168:897-905.

4. Khazardoost S, Maryamnoorzadeh, Abdollahi A, Shafaat M. Comparison of 8-h urine protein and random urinary protein-to-creatinine ratio with 24 -h urine protein in pregnancy. J Matern Fetal Neonatal Med 2012;25:138-140.

5. Robert M, Sepandj F, Liston RM, Dooley KC. Random protein-creatinine ratio for the quantitation of proteinuria in pregnancy. Obstet Gynecol 1997;90:893-895.

6. Yamasmit W, Wongkitisophon K, Charoenvidhya D, Uerpairojkit B, Chaithongwongwatthana S. Correlation between random urinary protein-to-creatinine ratio and quantitation of 24-hour proteinuria in preeclampsia. J Med Assoc Thai 2003;86:69-73.

7. Sibai BM. Treatment of hypertension in pregnant women. N Engl J Med 1996;335:257-265.

8. Chapter 1: definition and classification of CKD. Kidney Int Suppl (2011) 2013;3:19-62.

9. National Kidney Foundation. K/DOQI clinical practice guidelines for chronic kidney disease: evaluation, classification, and stratification. Am J Kidney Dis 2002;39(2 Suppl 1):S1-S266.

10. Qiu S. Clinical analysis of the outcome of pregnancy with chronic renal disease. Zhonghua Fu Chan Ke Za Zhi 1993;28:595-598.

11. Haas DM, Sabi F, McNamara M, Rivera-Alsina M. Comparing ambulatory spot urine protein/creatinine ratios and 24 -h urine protein measurements in normal pregnancies. J Matern Fetal Neonatal Med 2003;14:233-236.

12. Saudan PJ, Brown MA, Farrell T, Shaw L. Improved methods of assessing proteinuria in hypertensive pregnancy. Br J Obstet Gynaecol 1997;104:1159-1164.

13. Bell SC, Halligan AW, Martin A, et al. The role of observer error in antenatal dipstick proteinuria analysis. $\mathrm{Br} \mathrm{J}$ Obstet Gynaecol 1999;106:1177-1180.

14. Rizk DE, Agarwal MM, Pathan JY, Obineche EN. Predicting proteinuria in hypertensive pregnancies with urinary protein-creatinine or calcium-creatinine ratio. J Perinatol 2007;27:272-277.
15. Somanathan N, Farrell T, Galimberti A. A comparison between 24-hour and 2-hour urine collection for the determination of proteinuria. J Obstet Gynaecol 2003;23:378380.

16. Amirabi A, Danaii S. A comparison of 4- and 24-hour urine samples for the diagnosis of proteinuria in pregnancy. Iran J Med Sci 2011;36:167-171.

17. Park JH, Chung D, Cho HY, et al. Random urine protein/ creatinine ratio readily predicts proteinuria in preeclampsia. Obstet Gynecol Sci 2013;56:8-14.

18. Boler L, Zbella EA, Gleicher N. Quantitation of proteinuria in pregnancy by the use of single voided urine samples. Obstet Gynecol 1987;70:99-100.

19. Aggarwal N, Suri V, Soni S, Chopra V, Kohli HS. A prospective comparison of random urine protein-creatinine ratio vs 24 -hour urine protein in women with preeclampsia. Medscape J Med 2008;10:98.

20. Baba Y, Yamada T, Obata-Yasuoka M, et al. Urinary protein-to-creatinine ratio in pregnant women after dipstick testing: prospective observational study. BMC Pregnancy Childbirth 2015;15:331.

21. James DK, Steer PJ, Weiner CP, Gonik B. High Risk Pregnancy: Management Options. 3rd ed. Philadelphia: Saunders/Elsevier, 2006:772-777.

22. Gabbe SG, Niebyl JR, Simpson JL. Obstetrics: Normal and Problem Pregnancies. 5th ed. Philadelphia: Churchill Livingstone/Elsevier, 2007:863-865.

23. ACOG Committee on Practice Bulletins: Obstetrics. ACOG practice bulletin. Diagnosis and management of preeclampsia and eclampsia. Number 33, January 2002. Obstet Gynecol 2002;99:159-167.

24. Papanna R, Mann LK, Kouides RW, Glantz JC. Protein/ creatinine ratio in preeclampsia: a systematic review. Obstet Gynecol 2008;112:135-144.

25. Evans W, Lensmeyer JP, Kirby RS, Malnory ME, Broekhuizen FF. Two-hour urine collection for evaluating renal function correlates with 24-hour urine collection in pregnant patients. J Matern Fetal Med 2000;9:233-237.

26. Adelberg AM, Miller J, Doerzbacher M, Lambers DS. Correlation of quantitative protein measurements in 8-, 12-, and 24-hour urine samples for the diagnosis of preeclampsia. Am J Obstet Gynecol 2001;185:804-807. 